\title{
Dynamic Manipulation of Flexible Objects with Torque Sequence Using a Deep Neural Network
}

\author{
Kento Kawaharazuka ${ }^{1}$, Toru Ogawa ${ }^{2}$, Juntaro Tamura ${ }^{2}$, and Cota Nabeshima ${ }^{2}$
}

\begin{abstract}
For dynamic manipulation of flexible objects, we propose an acquisition method of a flexible object motion equation model using a deep neural network and a control method to realize a target state by calculating an optimized time-series joint torque command. By using the proposed method, any physics model of a target object is not needed, and the object can be controlled as intended. We applied this method to manipulations of a rigid object, a flexible object with and without environmental contact, and a cloth, and verified its effectiveness.
\end{abstract}

\section{INTRODUCTION}

Flexible object manipulation is one of the most challenging manipulations, and has been studied vigorously. This type of manipulation includes knotting a rope, folding a cloth, etc., and they are fundamental motions in our daily lives.

When we classify these manipulations from the viewpoint of static or dynamic manipulations and controls using physics-based modeling or machine learning, the studies have mainly focused on the static manipulation of flexible objects using physics-based modeling [1]. These methods estimate a geometrical relationship of an object, and determine the next movements to follow a target geometrical relationship [2], [3], [4], [5]. Meanwhile, several methods, which acquire flexible object manipulation from trial and error using a machine learning approach, have been developed [6], [7], [8]. These approaches do not need any physics models of a target object, and are versatile because the models are constructed from motion data. On the other hand, dynamic manipulations of flexible objects such as cloth folding, knotting, etc. have been studied mostly in physicsbased modeling approaches [9], [10]; nevertheless, there are a few studies using machine learning approaches, especially deep learning [11]. However, their target states are achieved by quasi-static movements, and they only use joint angles or joint velocities as the control input, though joint torques are critical in realizing dynamic movement. Also, with the spread of torque controlled robots [12], [13], [14], which can contact the environment softly, we should develop a dynamic manipulation method with torque command. In this study, to address these problems, we propose a dynamic control method of flexible objects to realize a target state from a current state using machine learning with joint torque command.

\footnotetext{
1 An author is associated with Department of Mechano-Informatics, Graduate School of Information Science and Technology, The University of Tokyo.kawaharazuka@jsk.t.u-tokyo.ac.jp

${ }^{2}$ Authors are associated with Preferred Networks, Inc. \{ogawa, tamura, cota\}@ preferred.jp
}

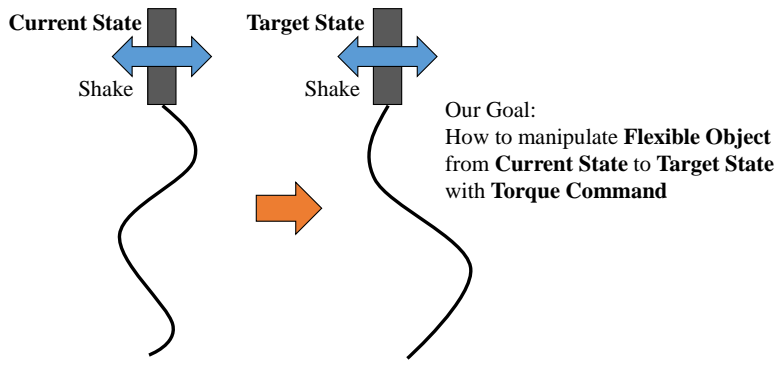

Fig. 1. Our goal in this study.

To realize real-time dynamic manipulation of flexible objects, we developed a system (Fig. 2) which has characteristics:

- Acquisition of flexible object motion equation model from motion data with random torque command using a deep neural network

- Calculation of optimized time-series torque command by backpropagation to the input torque command using the acquired motion equation model

- Generation of initial time-series torque commands before the backpropagation for real-time dynamic control

By using this system, we can move the flexible object from a current state to a target state dynamically with time-series torque command. The detailed contributions of this study are as shown below.

- A network structure representing motion equation using vision image and time-series torque command

- Real-time calculation process of optimized time-series torque command

- Comparison of task realization with changes of parameters in several experiments

In the following sections, at first, we introduce DynamicsNet, which represents the dynamic motion equation of a target object. Then, we explain the calculation method of optimized time-series torque command using this Dynamics-

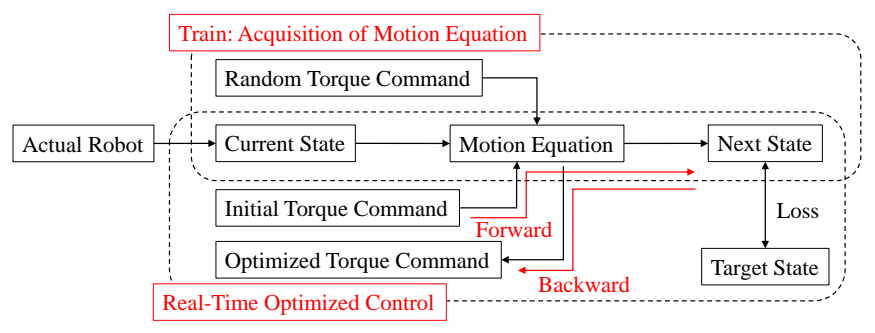

Fig. 2. System overview of this study. 


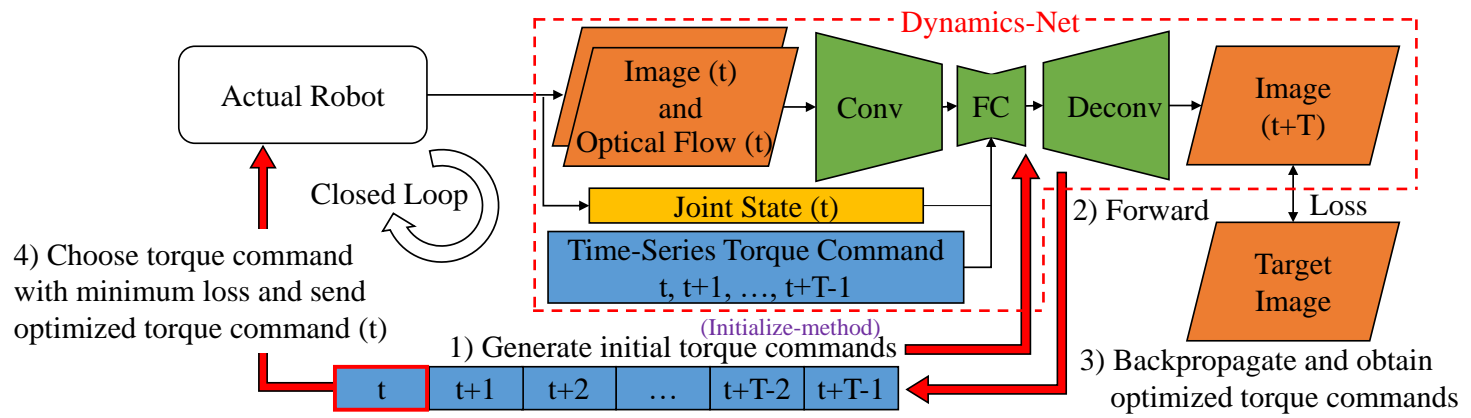

Fig. 3. Network structure of Dynamics-Net and calculation of optimized time-series torque command.

Net. Finally, we verify the effectiveness of this method by manipulation experiments of a rigid object, a flexible object with and without environmental contact, and a cloth.

\section{Dynamics-Net And OuR System}

\section{A. Dynamics-Net}

A general motion equation of robot manipulator can be formulated as,

$$
M(\boldsymbol{\theta}) \ddot{\boldsymbol{\theta}}+\boldsymbol{c}(\boldsymbol{\theta}, \dot{\boldsymbol{\theta}})+\boldsymbol{g}(\boldsymbol{\theta})=\boldsymbol{\tau}
$$

where $\boldsymbol{\theta}, \dot{\boldsymbol{\theta}}, \ddot{\boldsymbol{\theta}}$ are joint position, velocity, and acceleration respectively, $\boldsymbol{\tau}$ is joint torque, $M$ is an inertial matrix, $c$ expresses centrifugal force, Coriolis force, viscous friction, etc., and $\boldsymbol{g}$ is gravity torque. When regarding the structure of flexible objects as under-actuated multi-link structures, we can represent the motion equation of flexible objects as Eq. 1. This equation means that when we apply torque command $\tau$ to the object with its current posture $\boldsymbol{\theta}$ and velocity $\dot{\boldsymbol{\theta}}$, we can obtain acceleration $\ddot{\theta}$ at the next frame. Then, we can know the posture one frame or $T$ frames ahead, by integrating the acceleration. We represent the postures as images, since they can easily handle flexible objects, which have infinite degrees of freedom (DOFs). For the same reason, we use optical flow to represent the velocity.

Dynamics-Net is a network representing this equation using a deep neural network as shown in Fig. 3 First, the convolution layers extract the current image feature from the image and optical flow of the target object. Second, the fully connected layers merge the current image feature, joint states (current position, velocity, and torque of each actuator), and time-series torque command until after $T$ frames. The frame length of time-series torque command $T$ is a hyperparameter, and we discuss it in Section IV. Third, the deconvolution layers predict the final state, i.e., the image after $T$ frames, from the merged feature vector.

\section{B. Calculation of Target Torque Command}

We will explain how to calculate optimized time-series torque command to realize the target image of the flexible object using Dynamics-Net. The details are shown in Fig. 3 . and the process is as follows.

1) Generate initial time-series torque commands before the optimization of the torque commands

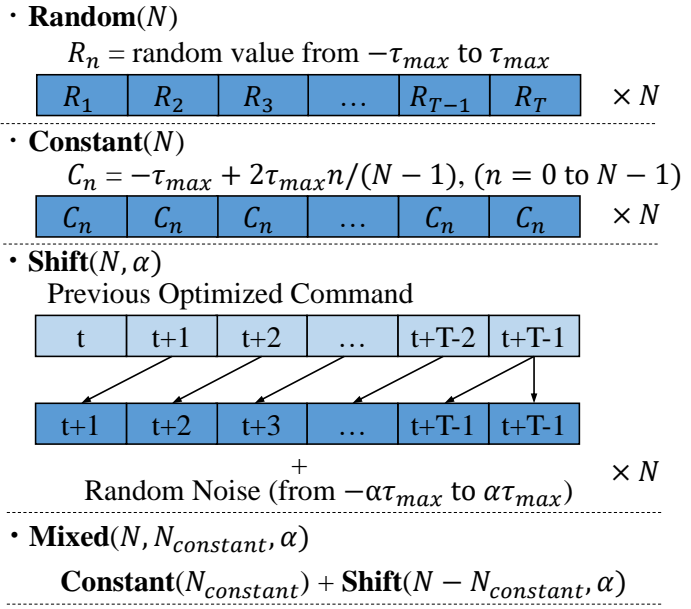

Fig. 4. The method to generate initial time-series torque commands before the optimization.

2) Obtain the current image and optical flow from the actual robot, feed them into Dynamics-Net with the initial time-series torque commands, and calculate the losses between the predicted and target images

3) Optimize the initial time-series torque commands by backpropagation of the losses

4) Choose the optimized torque command with the minimum loss, and send the torque command of the current frame as the target torque command

Next, we will explain the details of 1)-4).

First, 1) is the most controversial point in this study. We express the maximum joint torque as $\tau_{\max }$ and the minimum as $-\tau_{\max }$. Fig. 4 shows several generation methods of timeseries torque commands for Dynamics-Net, named Initializemethods. We define 4-type Initialize-methods:

- $\operatorname{Random}(N)$ : generate $N$ random time-series torque commands within $\left[-\tau_{\max }, \tau_{\max }\right]$

- $\operatorname{Constant}(N)$ : divide the torque limit $\left[-\tau_{\max }, \tau_{\max }\right]$ equally into $N$ parts and generate $N$ constant timeseries torque commands

- Shift $(N, \alpha)$ : shift the previous optimized timeseries torque command, add random noise within $\left[-\alpha \tau_{\text {max }}, \alpha \tau_{\text {max }}\right]$, and generate $N$ samples

- $\operatorname{Mixed}\left(N, N_{\text {constant }}, \alpha\right)$ : generate $N$ samples by adding $\operatorname{Constant}\left(N_{\text {constant }}\right)$ and $\operatorname{Shift}\left(N-N_{\text {constant }}, \alpha\right)$

We set $N=10, \alpha=0.25, N_{\text {constant }}=3$. Because the 
parameter $N$ depends on the control frequency $(30 \mathrm{~Hz}$ in this study) and computational resources, we do not discuss it much in this study. These Initialize-methods contribute the efficient search of time-series torque commands to realize the target image. Also, because several time-series torque commands realizing the same target image exist, we verify how these Initialize-methods contribute to the dynamic movement.

Regarding 2), the loss function is important. We use binary images without color information as input and output images. We use the loss with decreased sensitivity to posture deviations by blurring the target image, as shown below,

$$
\begin{aligned}
\boldsymbol{S}_{\text {target }}^{\prime} & =1.0-\tanh \left(\beta \cdot \mathrm{DT}\left(\boldsymbol{S}_{\text {target }}\right)\right) \\
\text { Loss } & =\operatorname{MSE}\left(\boldsymbol{S}_{\text {predicted }}-\boldsymbol{S}_{\text {target }}^{\prime}\right)
\end{aligned}
$$

where $\boldsymbol{S}_{\text {target }}$ is the target image, $\boldsymbol{S}_{\text {predicted }}$ is the predicted image, tanh is a hyperbolic tangent, DT (DistanceTransform) is the image of distances to the nearest object pixel at each pixel, $\beta$ is a scaling factor ( 0.5 in this study), and MSE is mean squared error.

Regarding 3) and 4), devising of the backward for realtime control and optimization of time-series torque command are important. At first, for real-time control, we remove the useless backward calculation of convolution layers. In addition, because the current image and optical flow are the same at the current frame, we feed them into the convolution layers just once. Next, we optimize the time-series torque command by backpropagation [15] like in [8], [16],

$$
\begin{aligned}
\boldsymbol{g} & =d \text { Loss } / d \boldsymbol{\tau}^{t s} \\
\boldsymbol{\tau}_{\text {optimized }}^{t s} & =\boldsymbol{\tau}_{\text {initial }}^{t s}-\gamma \boldsymbol{g} /|\boldsymbol{g}|
\end{aligned}
$$

where, $\boldsymbol{\tau}^{t s}$ expresses time-series torque command, $\boldsymbol{\tau}_{\text {initial }}^{\text {ts }}$ is the initial $\boldsymbol{\tau}^{t s}, \boldsymbol{\tau}_{\text {optimized }}^{t s}$ is the optimized $\boldsymbol{\tau}^{t s}, \gamma$ is a constant $\left(0.25 \tau_{\max }\right.$ in this study). $\boldsymbol{\tau}_{\text {initial }}^{\text {ts }}$ is constructed by $N$ samples, we choose one sample of $\tau_{\text {initial }}^{t s}$ with the minimum loss after forwarding, and obtain $\tau_{\text {optimized }}^{\text {ts }}$ by optimizing it. Then, we conduct the forwarding of DynamicsNet again by setting $\boldsymbol{\tau}_{\text {initial }}^{t s}$ as $\tau_{\text {optimized }}^{t s}$. As the target joint torque at this frame, we use $\tau_{\text {optimized }}^{\text {ts }}(t)$ if the calculated loss is smaller than the loss before optimization; otherwise, we use $\tau_{\text {initial }}^{t s}(t)$.

\section{Implementation Details}

Regarding image processing, because we basically treat 2D movements, we preprocess the image by seven steps: crop, resize, background subtraction, blurring, thresholding, closing, and opening. We extract the region of interest (ROI) by cropping, and resize it to the size of $64 \times 64$. Regarding the optical flow, we make a 2-channel image of the $\mathrm{x}$ - and $y$-axis of the optical flow.

Regarding the network structure of Dynamics-Net, as described in Subsection II-A, it consists of three blocks: Conv, FC, and Deconv. For Conv block, we use five convolution layers. Each layer has 4, 8, 16, 32, and 64 channels, respectively. The number of channels in the input layer is 3: image and optical flow ( $\mathrm{x}$ and $\mathrm{y}$ ). In all layers, the kernel
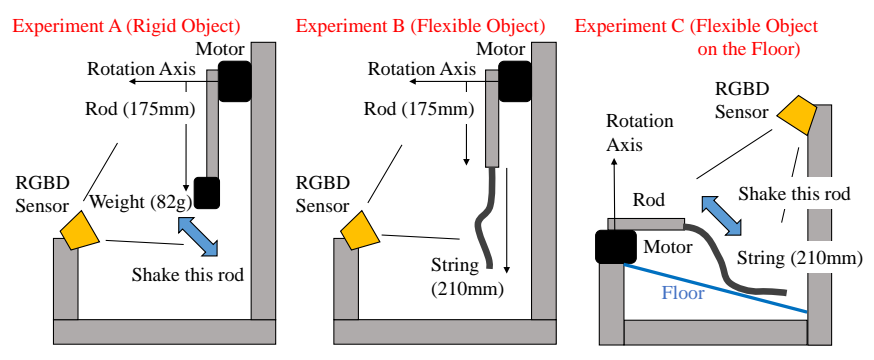

Fig. 5. Details of experimental setup A (rigid object), B (flexible object), and $\mathrm{C}$ (flexible object on the floor).

size is $3 \times 3$, stride is $2 \times 2$, and padding is 1 . In FC block with fully connected layers, the number of units are $128+M(3+T), 128,128,128$, and 256 , where $M$ is the number of actuators and $3+T$ means the joint states and time-series torque command of each actuator. Deconv block has deconvolution layers which are the same structures as Conv block. Batch normalization [17] is applied to each layer except the output layer. The activation function of each layer except the output layer is ReLU [18], and we normalize the output layer by sigmoid.

We implemented our network by Chainer [19], and conducted our whole processes without GPU.

\section{EXPERIMENTS}

We conducted four manipulation experiments (A-D). We evaluated our system quantitatively by manipulations of two fundamental objects: A) a rigid object and B) a flexible object. A) is the simplest setting bacause the target object has only one link, whereas the target object in B) has infinite DOFs and links. Also, we conducted two object manipulations in more practical situations: C) a flexible object on the floor and D) a cloth moving in 3D with 2 DOFs manipulator. In C), the object motion is affected by the environmental contact. In D), the motion equation is more complex and we have to control more than one actuators concurrently.

Fig. 5] shows the experimental settings of A-C. We cover these experimental setups by a black curtain. The actuator is XM430-W350-R (Dynamixel motor), and RGBD sensor is D435 (Intel Realsense). In A), we added weight to the tip of the rod, so the rod cannot be raised slowly and needs to be moved dynamically. In C), the string moves on the floor, so there is a frictional force. We describe the setting of D) in Subsection III-D separately.

As an image similarity metric, we used chamfer distance $d_{\text {chamfer }}[20]$ as shown below,

$$
d_{\text {chamfer }}\left(\boldsymbol{S}_{1}, \boldsymbol{S}_{2}\right)=\sum\left(\boldsymbol{S}_{1} \cdot \mathrm{DT}\left(\boldsymbol{S}_{2}\right)+\boldsymbol{S}_{2} \cdot \mathrm{DT}\left(\boldsymbol{S}_{1}\right)\right)
$$

where the unit of $d_{\text {chamfer }}$ is px. We set the current image as $\boldsymbol{S}_{1}$ and the target image as $\boldsymbol{S}_{2}$. The target image can change over time, but we set a constant image as the target image in this study.

\section{A. Rigid Object Manipulation}

At first, we collected 10,800 frames of motion data of rigid object manipulation by random torque commands for 

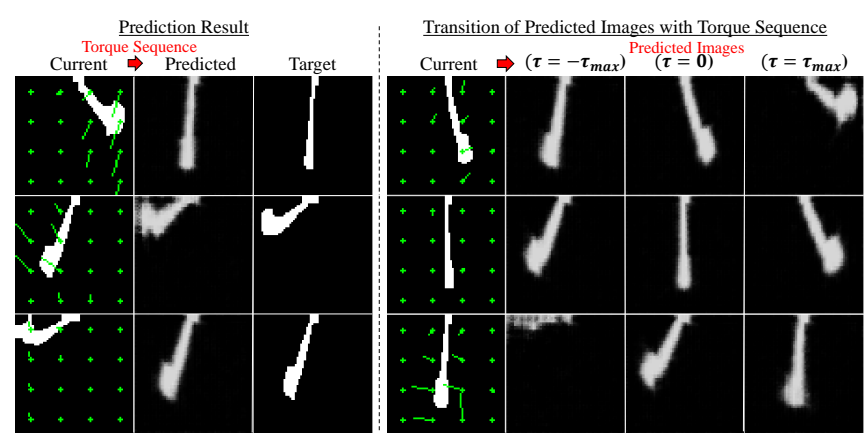

Fig. 6. Prediction result using the trained Dynamics-Net and transition of predicted images with time-series torque command, in rigid object experiment.

6 minutes, where we set $\tau_{\max }=0.2[\mathrm{Nm}]$ and $d \tau_{\max }=$ $0.1[\mathrm{Nm} /$ frame $]$. This data collection does not need any annotation and we only need to wait for a short time. Then, we trained Dynamics-Net using the data. We show the prediction results in the left figure of Fig. 6, where $T=10$ and the green lines express optical flow thinning out for visualization. We can see that Dynamics-Net can predict images after 10 frames properly and the predicted images are blurred due to the loss function of Eq. 2 which blurs target images. Next, we show the transition of predicted images by changing the timeseries torque command $\boldsymbol{\tau}^{t s}=-\boldsymbol{\tau}_{\text {max }}^{t s}, \mathbf{0}, \boldsymbol{\tau}_{\max }^{t s}$, respectively, in the right figure of Fig. 6 . We can see that the predicted images transition by the change of the torque command and they are reasonable from the current image and optical flow.

Finally, we conducted control experiments of a rigid object to realize the target image using Dynamics-Net. First, we show how the target images were realized by the proposed method in Fig. 7. We prepared Target A1 and Target A2 as target images. We set $T=10$ and used Mixed as Initializemethod. We can see the evaluation value $d_{\text {cham fer }}$ converged rapidly to about zero. Also, the target joint torque vibrated to keep the target image after the convergence. Second, we show the comparison of target image realization when changing $T$ to 5,10,15 and Initialize-method to Random, Constant, Shift, and Mixed, in Fig. 8 We conducted a 10-second experiment 5 times regarding each parameter from the state with the rod taken down, and measured the target image realization. The horizontal axis of each graph expresses the threshold of $d_{\text {chamfer }}\left(t_{\text {chamfer }}\right)$, and the vertical axis expresses the rate of frames below the

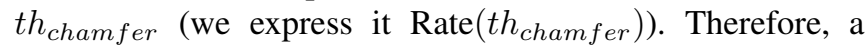
good parameter has higher Rate $\left(h_{\text {chamfer }}\right)$ when $t h_{\text {chamfer }}$ is low. No Optimization refers to the line of the graph using random torque control without any optimization. In Fig. 8 . with all parameters, our framework realized target images better than No Optimization, and $T=10$ is the best. Also, when $T=10$, Mixed can realize target images the best, and the second best is Constant.

\section{B. Flexible Object Manipulation}

We conducted a flexible object manipulation experiment, but the basic setting is the same as the rigid object manipulation. As with the rigid object experiment, we trained
Dynamics-Net from 10,800 motion data of random torque control of a flexible object. We show the prediction results in the left figure of Fig. 9 and the transition of predicted images by changing torque command in the right figure of Fig. 9 We can see that Dynamics-Net can predict images after 10 frames properly and the transition of predicted images is also reasonable. Then, we prepared Target B1 and B2 as target images, and show how target images were realized in Fig. 10. We set $T=10$ and used Constant regarding Target B1 and Shift regarding Target B2. We can see the evaluation value vibrated slowly, because we set images which cannot stand still as target images. Also, we show the comparison of target image realization when changing $T$ to $5,10,15$ and Initialize-method to Random, Constant, Shift, and Mixed, in Fig. 11. With all parameters, our framework realized target images better than No Optimization, and $T=10,15$ are better than $T=5$. Also, Constant and Mixed can realize Target B1 the best, and Shift can realize Target B2 the best.

\section{Flexible Object Manipulation on the Floor}

In this experiment, because environmental contact is added, motion generation using physics-based modeling is more difficult due to the difficulty of friction modeling. We prepared Target $\mathrm{C}$ as a target image, and set $T=10$ and Mixed as Initialize-method. The result is shown in Fig. 12 The predicted images realized approximately the Target $\mathrm{C}$, and the current images realized approximately the Target $\mathrm{C}$ afterwards. Therefore, Dynamics-Net can even acquire motion equation model with environmental forces such as frictional force.

\section{Cloth Manipulation}

We conducted cloth manipulation in 3D with a robot having 2 DOFs. The experimental setup is shown in the upper left figure of Fig. 13 Although we have handled 2D movements so far, to consider 3D movements, we changed the input layer of Dynamics-Net to 4 channels: current image, optical flow (x and y), and depth image. The values of the depth image are cropped and normalized to a value from 0 to 1 . Ideally, we must add the optical flow in the z-axis to the input layer and the depth image to the output layer, but we did not add these changes due to the difficulty of the loss function setting and increased computational complexity. The actuators are XM430-W210-R (Dynamixel motor). We set $\tau_{\max }=0.3[\mathrm{Nm}], T=10$, and Mixed as Initialize-method. We prepared Target $\mathrm{D}$ as a target image, and the folded cloth was unfolded properly by the proposed system, as shown in the right figure of Fig. 13 We show the feasibility to realize flexible object manipulation in 3D.

\section{Discussion}

First, regarding the rigid object experiment, the results show that the best is Mixed and the second best is Constant, but regarding the flexible object experiment, Shift is the best, especially in Target B2. From these results, we can consider that Constant is good at realizing static movements because it provides constant torques, and Shift is good at 

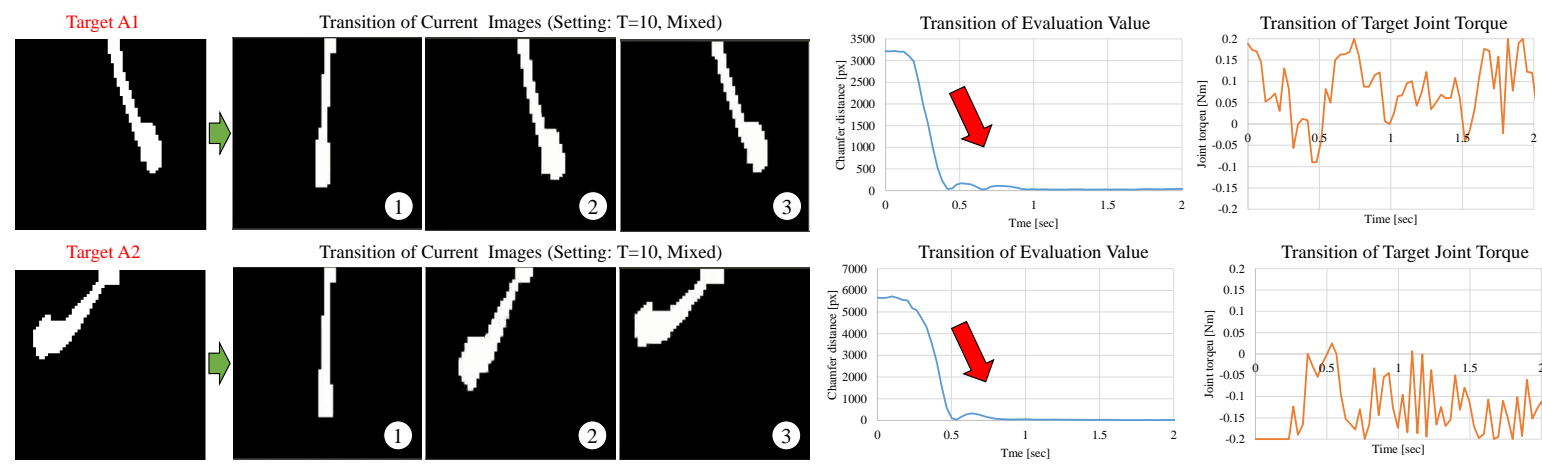

Fig. 7. Rigid object manipulation: the transition of current images, evaluation value, and target joint torque.
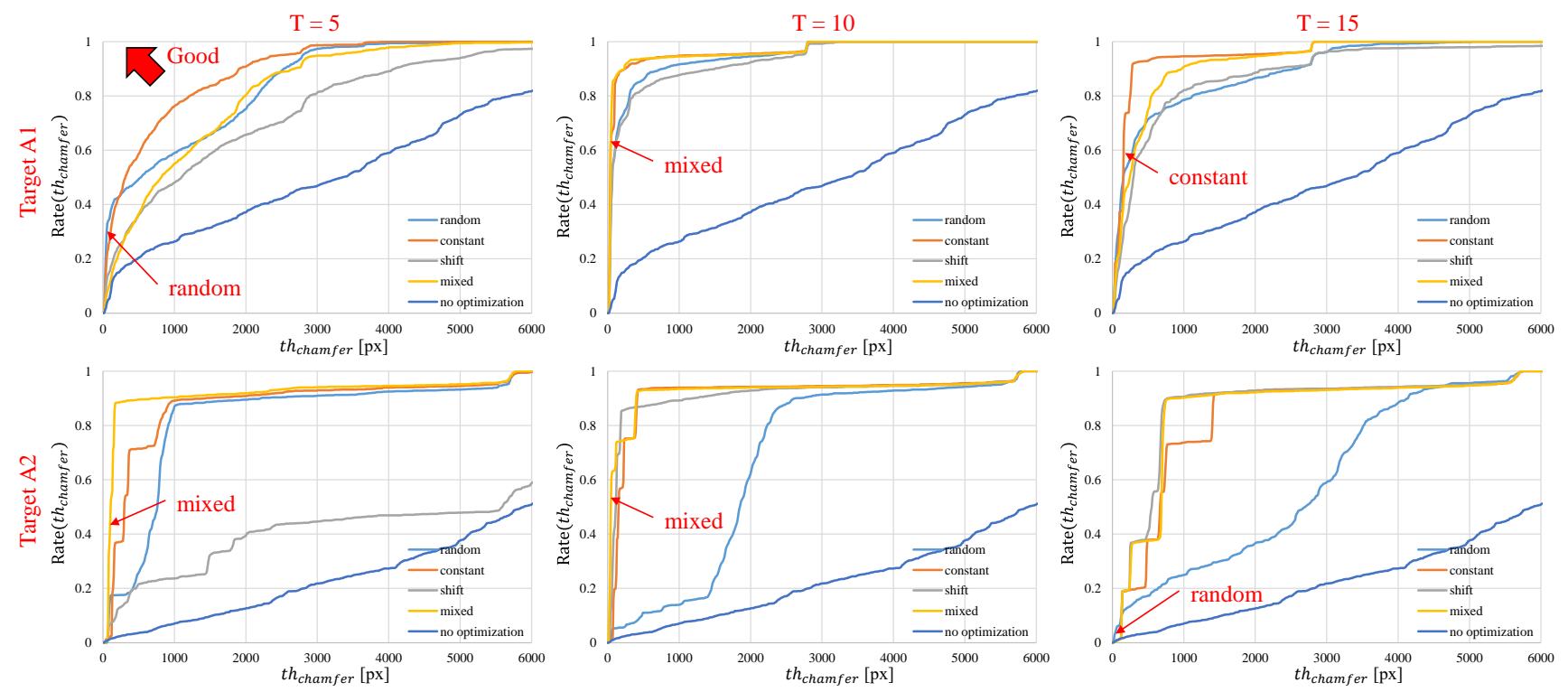

Fig. 8. The relationship between $t h_{\text {chamfer }}$ and rate of frames below the $t h_{c h a m f e r}$ : Rate $\left(t_{c h a m f e r}\right)$, when changing Initialize-methods, $T$, and target images, in rigid object manipulation.
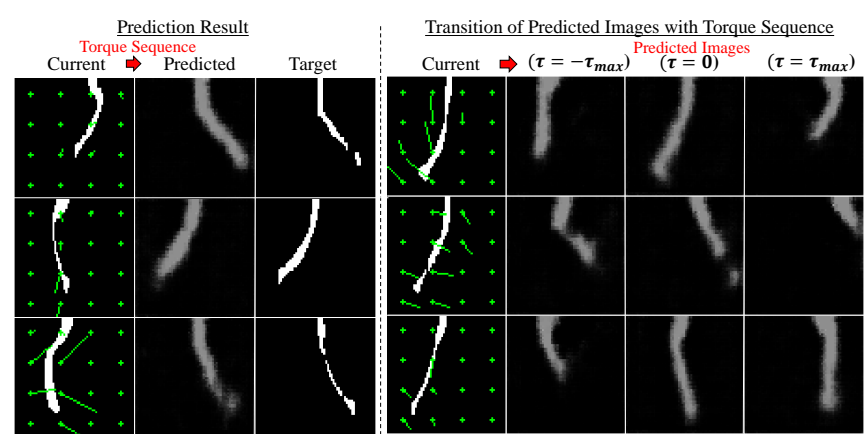

Fig. 9. Prediction result using the trained Dynamics-Net and transition of predicted images with time-series torque command, in flexible object experiment.

realizing dynamic movements because it considers the timeseries torque transition. In the rigid object experiment, we assume that Shift is effective approaching the target image, and Constant is effective at approximately the target image, so the target image was realized the best by using Mixed mixing Constant with Shift. On the other hand, in the flexible object experiment, especially Target B2 cannot be realized by static movements, so Mixed is not good for this target image, and Shift, which considers only time-series torque command transition, is the best.

Second, from the experimental results, we can see that $T$ should not be too small or too large. This is because we cannot realize target images far away when $T$ is too small, and we cannot obtain optimized torque command correctly, due to the difficulty of predicting the distant future, when $T$ is too large.

Third, the Dynamics-Net we implemented in this study is one of the simplest structures representing the motion equation, and we can consider other various structures. For example, we can insert recurrent structures like LSTM [21], or make the network structure lighter by using features extracted from AutoEncoder [22]. Also, we can adopt $T$ as a network input to use it as a variable, or output the images not only at $t+T$ but also at $[t+1, t+T]$. These changes may be able to consider the transition of current images and remove the hyperparameter $T$.

\section{CONCLUSION}

In this study, we proposed an acquisition method of a motion equation model of flexible object manipulation with 

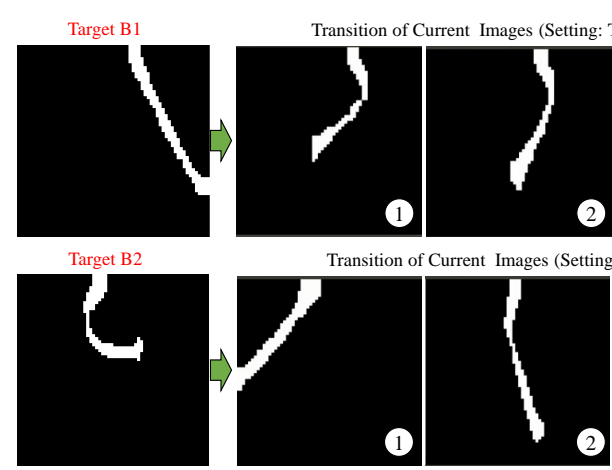

Transition of Current Images (Setting: T=10, Shift)
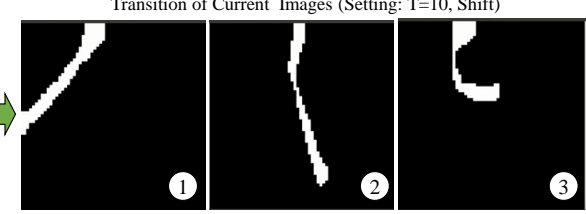

Fig. 10. Flexible object manipulation: the transition of current image, evaluation value, and target joint torque.
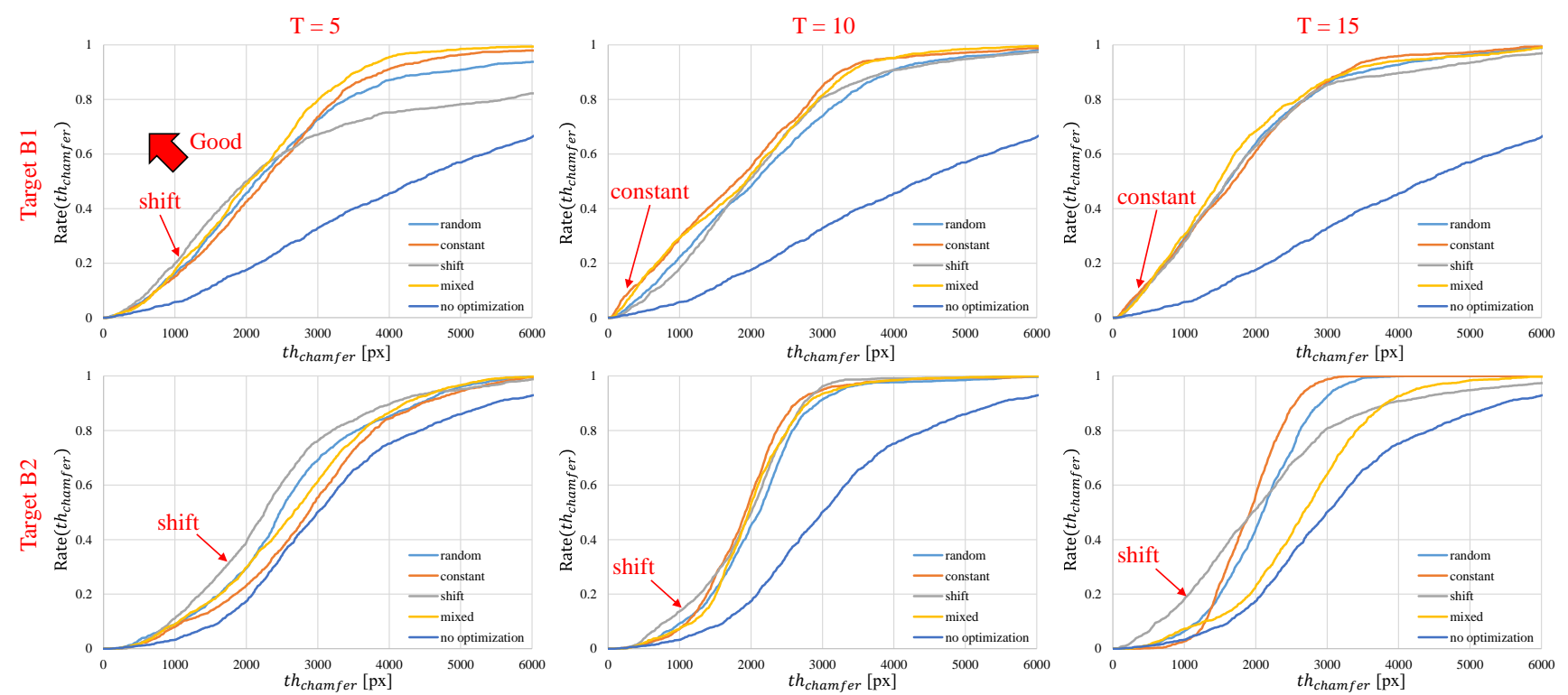

Fig. 11. The relationship between $t h_{c h a m f e r}$ and rate of frames below the $t h_{c h a m f e r}$ : Rate $\left(h_{c h a m f e r}\right)$, when changing Initialize-methods, $T$, and target images, in flexible object manipulation.

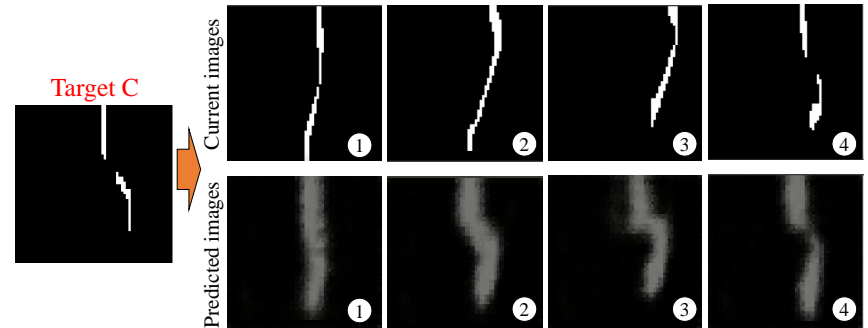

Fig. 12. Flexible object manipulation on the floor: the transition of current and predicted images.

torque command by Dynamics-Net and a calculation method of optimized time-series torque command to realize a target state. Dynamics-Net works as the motion equation of flexible object manipulation whose inputs are the current image, optical flow, joint states, and time-series torque command, and whose output is the predicted image after $T$ frames. We also investigated the calculation method of optimized timeseries torque command by backpropagation to inputs and the generation method of initial time-series torque commands for dynamic real-time control. Then we validated the effectiveness by experiments using a rigid object, a flexible object,

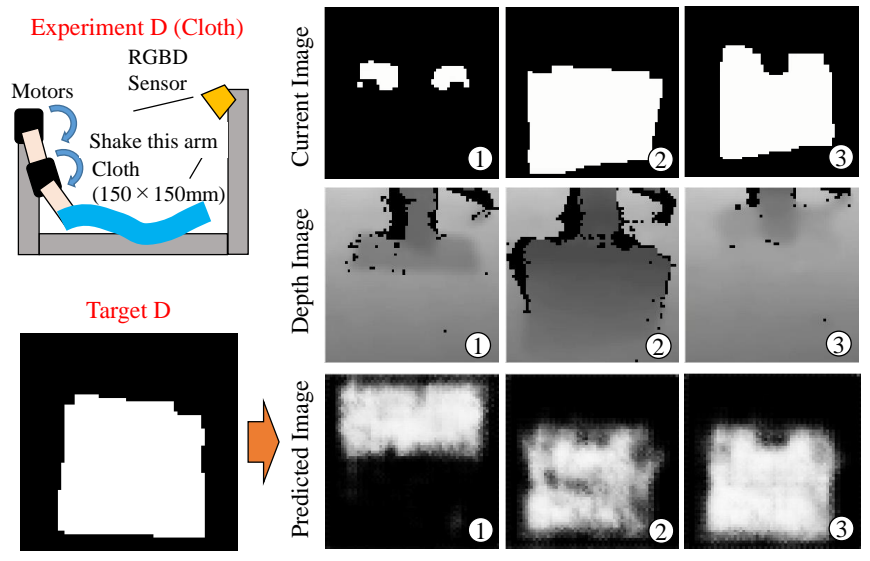

Fig. 13. Cloth manipulation in 3D: the experimental setup, and the transition of current, depth, predicted images.

a flexible object on the floor, and a cloth. In future works, we would like to validate the effectiveness of DynamicsNet when using a multi-DOFs manipulator, and apply this method to more practical tasks. 


\section{REFERENCES}

[1] P Jiménez. Survey on model-based manipulation planning of deformable objects. Robotics and computer-integrated manufacturing, Vol. 28, No. 2, pp. 154-163, 2012.

[2] M. Inaba and H. Inoue. Rope handling by a robot with visual feedback. Advanced Robotics, Vol. 2, No. 1, pp. 39-54, 1987.

[3] M. Saha and P. Isto. Manipulation Planning for Deformable Linear Objects. IEEE Transactions on Robotics, Vol. 23, No. 6, pp. 11411150, 2007.

[4] C. Elbrechter, R. Haschke, and H. Ritter. Folding paper with anthropomorphic robot hands using real-time physics-based modeling. In 2012 IEEE-RAS International Conference on Humanoid Robots, pp. 210-215, 2012.

[5] T. M. Caldwell, D. Coleman, and N. Correll. Optimal parameter identification for discrete mechanical systems with application to flexible object manipulation. In 2014 IEEE/RSJ International Conference on Intelligent Robots and Systems, pp. 898-905, 2014.

[6] A. X. Lee, H. Lu, A. Gupta, S. Levine, and P. Abbeel. Learning force-based manipulation of deformable objects from multiple demonstrations. In 2015 IEEE International Conference on Robotics and Automation, pp. 177-184, 2015.

[7] Z. Hu, P. Sun, and J. Pan. Three-Dimensional Deformable Object Manipulation Using Fast Online Gaussian Process Regression. IEEE Robotics and Automation Letters, Vol. 3, No. 2, pp. 979-986, 2018.

[8] D. Tanaka, S. Arnold, and K. Yamazaki. EMD Net: An EncodeManipulate-Decode Network for Cloth Manipulation. IEEE Robotics and Automation Letters, Vol. 3, No. 3, pp. 1771-1778, 2018.

[9] Y. Yamakawa, A. Namiki, and M. Ishikawa. Motion planning for dynamic knotting of a flexible rope with a high-speed robot arm. In 2010 IEEE/RSJ International Conference on Intelligent Robots and Systems, pp. 49-54, 2010.

[10] Y. Yamakawa, A. Namiki, and M. Ishikawa. Motion planning for dynamic folding of a cloth with two high-speed robot hands and two high-speed sliders. In 2011 IEEE International Conference on Robotics and Automation, pp. 5486-5491, 2011.

[11] P. Yang, K. Sasaki, K. Suzuki, K. Kase, S. Sugano, and T. Ogata. Repeatable Folding Task by Humanoid Robot Worker Using Deep Learning. IEEE Robotics and Automation Letters, Vol. 2, No. 2, pp. 397-403, 2017.

[12] J. Englsberger, A. Werner, C. Ott, B. Henze, M. A. Roa, G. Garofalo, R. Burger, A. Beyer, O. Eiberger, K. Schmid, and A. Albu-Schäffer. Overview of the torque-controlled humanoid robot TORO. In 2014 IEEE-RAS International Conference on Humanoid Robots, pp. 916923, 2014.

[13] S. Hyon, D. Suewaka, Y. Torii, and N. Oku. Design and Experimental Evaluation of a Fast Torque-Controlled Hydraulic Humanoid Robot. IEEE/ASME Transactions on Mechatronics, Vol. 22, No. 2, pp. 623634, 2017.

[14] C. Semini, V. Barasuol, J. Goldsmith, M. Frigerio, M. Focchi, Y. Gao, and D. G. Caldwell. Design of the Hydraulically Actuated, TorqueControlled Quadruped Robot HyQ2Max. IEEE/ASME Transactions on Mechatronics, Vol. 22, No. 2, pp. 635-646, 2017.

[15] D. E. Rumelhart, G. E. Hinton, and R. J. Williams. Learning representations by back-propagating errors. nature, Vol. 323, No. 6088, pp. 533-536, 1986.

[16] A. Byravan, F. Leeb, F. Meier, and D. Fox. SE3-Pose-Nets: Structured Deep Dynamics Models for Visuomotor Planning and Control. In 2018 IEEE International Conference on Robotics and Automation, pp. 3339-3346, 2018.

[17] S. Ioffe and C. Szegedy. Batch Normalization: Accelerating Deep Network Training by Reducing Internal Covariate Shift. In Proceedings of the 32nd International Conference on International Conference on Machine Learning, pp. 448-456, 2015.

[18] V. Nair and G. E. Hinton. Rectified linear units improve restricted boltzmann machines. In Proceedings of the 27th International Conference on International Conference on Machine Learning, pp. 807-814, 2010.

[19] S. Tokui, K. Oono, S. Hido, and J. Clayton. Chainer: a next-generation open source framework for deep learning. In Proceedings of Workshop on Machine Learning Systems in The 29th Annual Conference on Neural Information Processing Systems, 2015.

[20] G. Borgefors. Hierarchical chamfer matching: a parametric edge matching algorithm. IEEE Transactions on Pattern Analysis and Machine Intelligence, Vol. 10, No. 6, pp. 849-865, 1988.
[21] S. Hochreiter and J. Schmidhuber. Long short-term memory. Neural computation, Vol. 9, No. 8, pp. 1735-1780, 1997.

[22] G. E. Hinton and R. R. Salakhutdinov. Reducing the Dimensionality of Data with Neural Networks. Science, Vol. 313, No. 5786, pp. 504507, 2006. 